\title{
THEORETICAL AND EXPERIMENTAL METHODS TO SELECT AIRCRAFT HANDLING QUALITIES
}

\section{E. Zaichik, Y.P. Yashin, V.S. Perebatov, and P. A. Desyatnik}

\author{
TsAGI \\ Zhukovsky, Moscow Region, Russia
}

\begin{abstract}
A theoretical-experimental method is developed to analyze and adequately select aircraft handling qualities (HQ). A review is presented of the criteria developed by the authors to estimate the role of motion cues in controlling of an aircraft, and criteria to estimate the on-ground simulation fidelity. The method is presented to translate on-ground simulation results into real flight conditions.
\end{abstract}

\section{INTRODUCTION}

The selection of aircraft HQ is a very important issue which determines the flight safety. At the same time, developing of general methods capable to assess HQ for various aircraft characteristics is an extremely difficult problem.

One of the main methods to select aircraft HQ is simulator experiments. But simulator results often considerably differ from those received in flight (see, for example, [1]). It means that specific forces and angular accelerations significantly affect the controlling of an aircraft, and the difference between on-ground and in-flight results is determined by the lack of motion cues (angular accelerations and linear specific forces) or their inadequate reproduction on ground-based simulators.

Modern simulators allow reliable reproduction of all essential flight conditions except for motion cues. In recent years, motion fidelity has been improved by refining the technical means of motion cues reproduction. In some respects, the technical characteristics of motion systems have almost approached their perfection. Nevertheless, even the most sophisticated flight simulators can have low fidelity of motion cues reproduction. Thus, the motion cueing problem becomes more scientific than technical.

The key problem in simulation fidelity assessment is the simulation fidelity definition. According to Hess and Siwakosit [2], simulation fidelity can be defined 
as "the degree to which characteristics of perceivable states induce adequate pilot psychomotor and cognitive behavior for a given task and environment."

Different simulation fidelity measures are used by different researchers. Sometimes, pilot models are used. Of course, motion cues perception peculiarities are essential for selecting drive algorithms [3], but the role of pilot models as a simulation fidelity measure is sometimes overestimated. The fact is that the motion cues perception cannot be regarded as simulation fidelity measure, since not pilot's sensations as such are important, but according to the definition Hess and Siwakosit, the agreement between his "psychomotor and cognitive behavior" on ground and in flight.

The accuracy of piloting is very often used as an objective measure of motion fidelity. But such data (piloting accuracy, for example) do not take into account pilot's workload and, thus, fail to fully characterize the motion fidelity. A pilot's workload can differ greatly in on-ground and in-flight tests, while his piloting precision may not change thanks to his adaptation.

Pilot's opinions and rating scales, such as Sinacori's scale, or HQ rating scales like Cooper-Harper scale, are used as integral subjective measure of motion fidelity. But their absolute values give no answer on how far we are from the real flight having this or that motion system drive algorithms.

Thus, there is needed a certain parameter which would allow to estimate the degree of difference between the real flight and simulated conditions. This parameter can be, for example, the relative Cooper-Harper pilot ratings (expressed as percentages):

$$
\Delta \mathrm{PR}_{\text {relative }}=\frac{\mathrm{PR}_{\text {fixed }}-\mathrm{PR}_{\text {motion }}}{\mathrm{PR}_{\text {fixed }}-\mathrm{PR}_{\text {flight }}} .
$$

To use this motion fidelity measure, one should know, first, the in-flight pilot ratings $\mathrm{PR}_{\text {flight }}$, or, in other words, how motion cues affect the control of an aircraft. Second, one must know $\mathrm{PR}_{\text {motion, }}$, or, in other words, how the distortions arising due to drive algorithms can distort the pilot ratings as compared to inflight ratings.

It is impossible to study experimentally all the variety of aircraft parameters and flight conditions. Only theoretical methods can help to estimate the effect of motion cues. The existing theoretical methods (control anticipation parameter, bandwidth, Neal-Smith criteria, etc.) of handling qualities analysis do not allow estimating this effect not only in degree, but sometimes even in kind. For example, in accordance with the modern theoretical view, the best roll handling qualities are achieved at roll mode time constant values about zero, whereas in real flight, these values cause negative pilot opinion due to high-frequency accelerations arising [1].

Recently, TsAGI experts developed a theoretical approach to motion cues effect analysis [4]. The approach was then successfully applied to estimate the role of motion cues in roll [5], pitch [6], and yaw [7] control. Based on this ap- 
proach, an experimental method was developed to estimate the motion fidelity of on-ground simulations. Together, the theoretical approach and the experimental method, form an integral theoretical-experimental method to select aircraft HQ. The presented paper is addressed to this method discussion.

\section{THEORETICAL APPROACH TO ESTIMATE THE EFFECT OF MOTION CUES}

The main reasons for the differences between pilot ratings received in-flight and on fixed-base simulators are the lack of motion cues and the hazard factor (the factor of dangerousness). Neither one nor the other can be exactly reproduced on ground. Among these two reasons, the lack of motion cues is more significant in terms of the pilot ratings difference.

To estimate the effect of motion cues on pilot ratings, a special approach has been developed [4-7].

It is well-known that motion cues affect piloting beneficially in one cases and negatively in other cases. On one hand, the motion cues are the information factor, that is, they give the pilot some useful information of aircraft motion (Fig. 1). On the other hand, angular and linear accelerations are the physiological

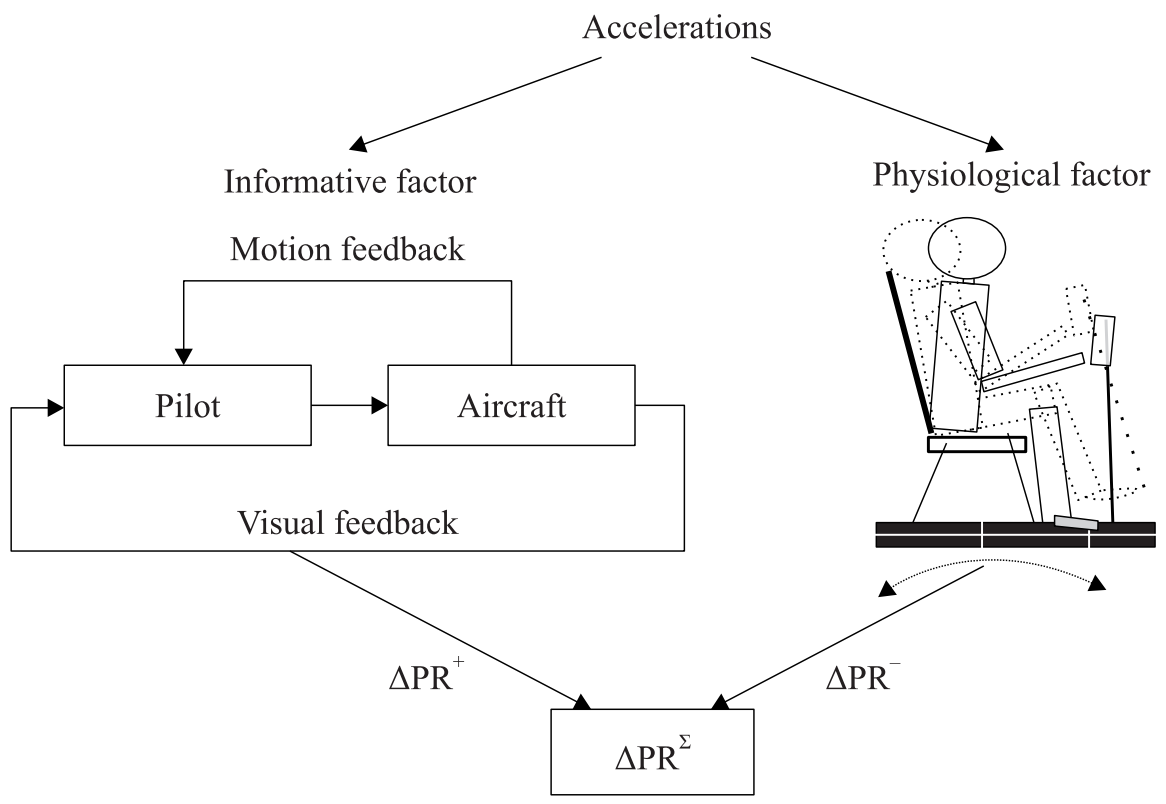

Figure 1 Acceleration roles in piloting 
factors as they cause body displacements which may be felt negatively by the pilot. The negative or beneficial role of motion cues in each case depends on which of the factors is dominating.

The degree of motion cues effect is assessed according to the difference in pilot ratings between on-ground simulation results and in-flight data $\Delta \mathrm{PR}$ :

$$
\Delta \mathrm{PR}=\mathrm{Pr}_{\text {flight }}-\mathrm{PR}_{\text {fixed }}
$$

where $\mathrm{PR}_{\text {flight }}$ is the in-flight pilot ratings and $\mathrm{PR}_{\text {fixed }}$ is the fixed-base simulation pilot ratings.

Since motion cues effect depends on the two factors (informative and physiological) simultaneously, $\Delta \mathrm{PR}$ can be written as follows:

$$
\Delta \mathrm{PR}=\mathrm{PR}^{-}-\mathrm{PR}^{+}
$$

where $\Delta \mathrm{PR}^{-}$and $\Delta \mathrm{PR}^{+}$are the pilot rating increments due to negative and beneficial effects, respectively.

The degree and the nature of the acceleration effect depend on aircraft characteristics, the type of piloting task, and the control axis.

\subsection{Beneficial Effect of Motion Cues}

Motion cues effect depends on the piloting task, motion cues intensity, and aircraft dynamics. According to [4], the motion cues effect is beneficial if two conditions are satisfied: $(i)$ the motion cues exceed their perception threshold; and $(i i)$ the motion cues are the second derivative of the visually controlled parameter:

$$
a=\ddot{v} .
$$

Piloting task. For roll disturbance task, the motion cues lead to visual cues and, unlike visual cues, motion cues are perceived regardless of pilot attention. Motion cues promote a reduction in the pilot's pure time delay which increases the pilot-aircraft system stability, widens bandwidth, and allows a pilot to increase his gain. As a result, the quality of control is better and pilot ratings improve $[3,4]$.

If the motion cues considerably deviate from the second derivative of the visually controlled parameter (for example, normal specific forces are not the second derivative of the visually controlled pitch), they do not affect piloting beneficially.

Motion cues intensity. It is obvious that accelerations do not have any effect on piloting if their maximum values are below sensitivity thresholds. If accelerations considerably exceed their thresholds $p \gg p_{\text {th }}$, variation in acceleration intensity does not change pilot ratings. In between, the beneficial pilot 
rating increment can be estimated with function $\Delta \mathrm{PR}^{+}=k\left(p_{\max }\right) \Delta \mathrm{PR}_{p_{\max } \gg p_{\text {th }}}$ where $k(p)$ can be estimated according to the function

$$
k(p)= \begin{cases}0, & p<p_{\mathrm{th}} \\ \frac{p-p_{\mathrm{th}}}{2 p_{\mathrm{th}}}, & p_{\mathrm{th}} \leq p \leq 3 p_{\mathrm{th}} \\ 1, & p>3 p_{\mathrm{th}}\end{cases}
$$

where $p$ and $p_{\text {th }}$ are the level of motion cues and their threshold level, respectively, and $\Delta \mathrm{PR}_{p \gg p_{\mathrm{th}}}$ is the pilot rating improvement increment when $p>3 p_{\mathrm{th}}$.

If conditions (1) and (2) are satisfied, the beneficial effect can be estimated in accordance with the following criterion:

$$
\Delta \mathrm{PR}^{+}=\frac{2}{1+\left(0.3 \omega_{\mathrm{BW}}\right)^{4}}
$$

where $\omega_{\mathrm{BW}}$ is the aircraft bandwidth which is determined as

$$
\varphi\left[\frac{\phi}{\delta_{\mathrm{as}}}\left(j \omega_{\mathrm{BW}}\right)\right]=-135^{\circ} .
$$

\subsection{Negative Effect of Motion Cues}

It is well known that even considerable $g$-loads are not perceived by the pilot as negative if they are deliberately created, while even barely perceived specific forces can be seen as unpleasant if arising uncontrollably. The latter type of specific forces is $(i)$ those arising due to the turbulence and ( $i i)$ high-frequency specific forces due to angular accelerations or due to aircraft structural elasticity.

Due to the difference in the frequency spectrums, the perception of specific forces of the 1st and 2nd types is different. Pilot's attitude to these two types of specific forces also differs since the specific forces due to the turbulence arise uncontrollably, while high-frequency specific forces of the second type are the aircraft response to the high-frequency component of pilot activities. Thus, the approaches to assess the effect of these two types of specific forces are also different.

Specific forces due to turbulence. A pilot does not compensate for the accelerations caused by turbulence; that is why, the magnitude of these accelerations assessed during fixed-base simulations practically equals their magnitude in real flight or in a moving-base simulations, provided there is adequate turbulence and aircraft dynamics models. Thus, pilot rating worsening due to the turbulence accelerations effect can be well assessed in fixed-base experiments. The data available show that the error in handling quality ratings due to the lack of lateral specific forces in fixed-base experiments can be approximately assessed from the curve in Fig. 2 or from the following expression: 


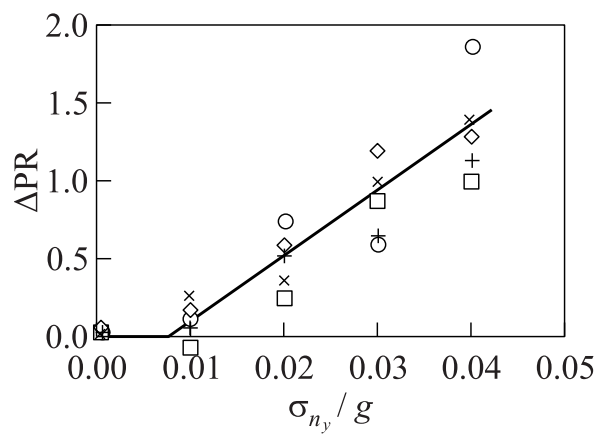

Figure 2 Effect of lateral turbulence on pilot ratings: signs refer to different $\tau_{R}$; and curve refers to criterion (4)

$$
\begin{aligned}
\Delta \mathrm{PR}^{-}\left(\sigma_{n_{y}}\right)=\operatorname{PR}\left(\sigma_{n_{y} \text { motion }}\right)-\operatorname{PR}\left(\sigma_{n_{y} \text { fixed }}\right) & \\
& = \begin{cases}0, & \sigma_{n_{y}}<0.008 \\
40 \sigma_{n_{y}}-0.3, & \sigma_{n_{y}} \geq 0.008 .\end{cases}
\end{aligned}
$$

Here, $\sigma_{n_{y}}$ is the root-mean-square (RMS) of lateral turbulence accelerations received in fixed-base experiments.

High-frequency accelerations due to pilot activity. The high-frequency unpleasant accelerations are the aircraft response to a high-frequency component in the pilot activity. The phenomenon is called aircraft abrupt response (AR). In [5-7], the criteria to estimate and predict the phenomenon in roll, pitch, and yaw were developed. Let consider the main principles of the approach by an example of roll.

The cause of unpleasant high-frequency linear accelerations is demonstrated in Fig. 3. This is frequency responses of aircraft in roll. It is seen that magnitude of aircraft response for $\tau_{R}=0.1 \mathrm{~s}$ at high frequencies is approximately 10 times as great as that for $\tau_{R}=1 \mathrm{~s}$. That is why, an aircraft responds to the highfrequency component in pilot's activities if the values of $\tau_{R}$ are small. As a result, high-frequency specific forces arise, which cause pilot's negative opinion of aircraft handling qualities.

The level of high-frequency accelerations typical for piloting depends not only on roll mode time constant, but on control sensitivity characteristics, aircraft structural elasticity, the pilot's position relative to the rotation axis, and some other characteristics.

As lateral accelerations arise as a result of aircraft angular motion, they are perceived by the pilot through angular motion. That is why, it is necessary to take into account the effect of angular motion on lateral accelerations perception. 

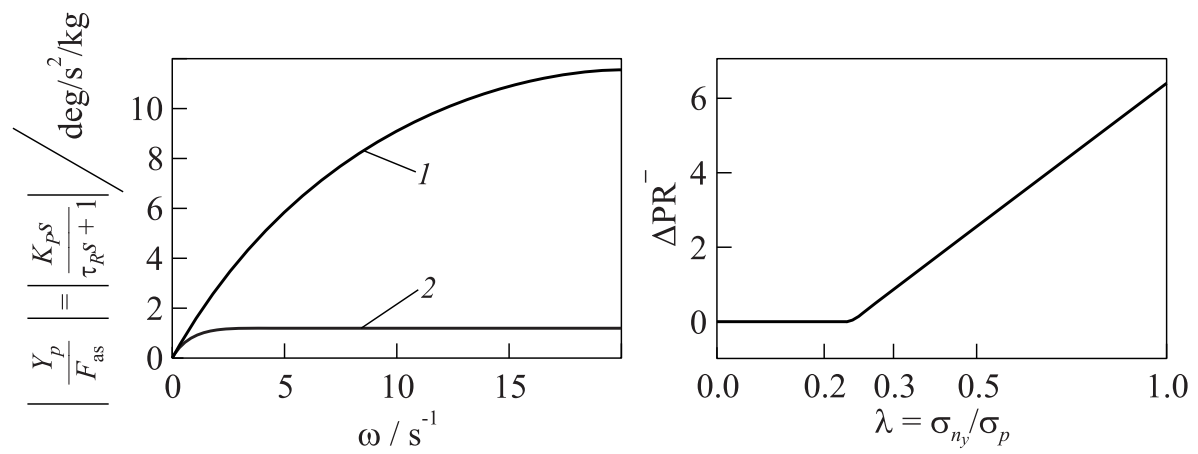

Figure 3 Frequency responses for roll ac- Figure 4 Criterion for estimation of celerations $\left(K_{P}=\right.$ const $)$ for different $\tau_{R}$ : abrupt response $1-0.1 \mathrm{~s}$ and $2-1 \mathrm{~s}$

As it was shown in [8], lateral acceleration sensitivity thresholds increase in proportion to roll rate amplitude. This made the basis to assume that the degree of lateral acceleration negative effect is determined by the ratio between the high-frequency lateral acceleration and roll rate $(\lambda)$ :

$$
\lambda=\frac{\sigma_{n_{y}}}{\sigma_{p}}
$$

where $\sigma_{n_{y}}$ and $\sigma_{p}$ are RMS of lateral specific forces in the cockpit and angular rates.

The degree of negative effect $\Delta \mathrm{PR}^{-}$as a function of $\lambda$ can be estimated according to the curve in Fig. 4 or to the following expression:

$$
\Delta \mathrm{PR}(\lambda)= \begin{cases}0, & \lambda<0.2 \\ 9 \log \lambda+6.3, & \lambda \geq 0.2\end{cases}
$$

In general case, $\sigma_{n_{y}}$ and $\sigma_{p}$ can be received as follows:

$$
\left.\begin{array}{rl}
\sigma_{n_{y}}^{2} & =\frac{1}{2 \pi} \int_{-\infty}^{+\infty}\left|Y_{n_{y} / \delta_{\mathrm{as}}}(j \omega) Y_{\mathrm{pilot}}(j \omega)\right|^{2} d \omega ; \\
\sigma_{p}^{2} & =\frac{1}{2 \pi} \int_{-\infty}^{+\infty}\left|Y_{p / \delta_{\mathrm{as}}}(j \omega) Y_{\text {pilot }}(j \omega)\right|^{2} d \omega
\end{array}\right\}
$$

where $Y_{n_{y} / \delta_{\text {as }}}(j \omega)$ and $Y_{p / \delta_{\text {as }}}(j \omega)$ are the transfer functions from roll stick displacements to lateral accelerations in the cockpit and to roll rates, respectively; and $Y_{\text {pilot }}$ is the pilot describing function. 
Pilot describing function has been chosen to meet the conditions stated in $[9$, 10] for the pilot performing sinusoidal stick deflections. As a result, the model of pilot activities was presented as white noise passing through the first-order filter:

$$
Y_{\text {pilot }}=\frac{1}{s+\omega_{c}\left(\delta_{p}^{*} / \delta_{p}\right)}
$$

where $\delta_{p}$ is the roll control sensitivity characteristics and $\delta_{p}^{*}$ is its certain "characteristic" value.

The considered approach to AR analysis is common for all control axes. Some deviations in mathematical expressions of the approach (5)-(8) are caused by peculiarities of piloting in particular control axes. The identification of the mathematics for the pitch and yaw control are given, respectively, in [6] and [7].

\section{METHOD TO CORRECT ON-GROUND SIMULATION RESULTS}

\subsection{Method to Correct Fixed-Base Handling Qualities Results}

Fixed-base pilot ratings $\mathrm{PR}_{\text {fixed }}$ are corrected as follows:

$$
\mathrm{PR}_{\text {flight }}=\mathrm{PR}_{\text {fixed }}+\Delta \mathrm{PR}^{-}-\Delta \mathrm{PR}^{+}
$$

where $\mathrm{PR}_{\text {flight }}$ is the in-flight pilot ratings and $\Delta \mathrm{PR}^{-}$and $\Delta \mathrm{PR}^{+}$are the pilot ratings increments for the negative and beneficial acceleration effects, respectively.

Note:

$$
\mathrm{PR}_{\text {flight }}= \begin{cases}1, & \text { if } \mathrm{PR}_{\text {fixed }}+\Delta \mathrm{PR}^{-}-\Delta \mathrm{PR}^{+} \leq 1 \\ 10, & \text { if } \mathrm{PR}_{\text {fixed }}+\Delta \mathrm{PR}^{-}-\Delta \mathrm{PR}^{+} \geq 10\end{cases}
$$

Beneficial acceleration effect $\Delta \mathrm{PR}^{+}$is estimated according to criterion (3).

Negative acceleration effect $\Delta \mathrm{PR}^{-}$for the case of aircraft $\mathrm{AR}$ is estimated according to criterion (5).

The data in Figs. 5-7 confirm the method reliability. Figure 5 compares HQ Levels received in real flight with that received in fixed-base simulations for different roll mode time constant $\tau_{R}$ and lateral control sensitivity $L_{F \text { as }}$ [1]. It is seen that the areas of good HQ received on ground and in flight do not even cross. Level 1 and Level 3 seem to switch places in the figure. The reason for this difference was lack of motion cues in on-ground simulations. In Fig. 5, there are also shown the estimations made according to the method above. It is seen that the estimations are in a good agreement with the empirical data both qualitatively and quantitatively. 


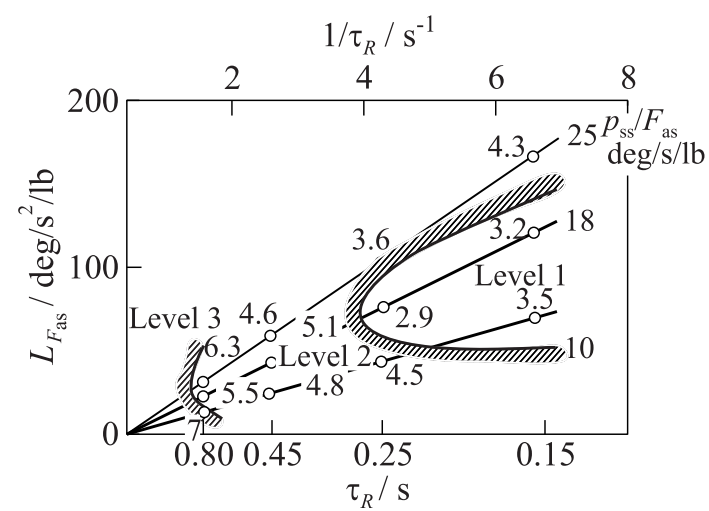

(a)

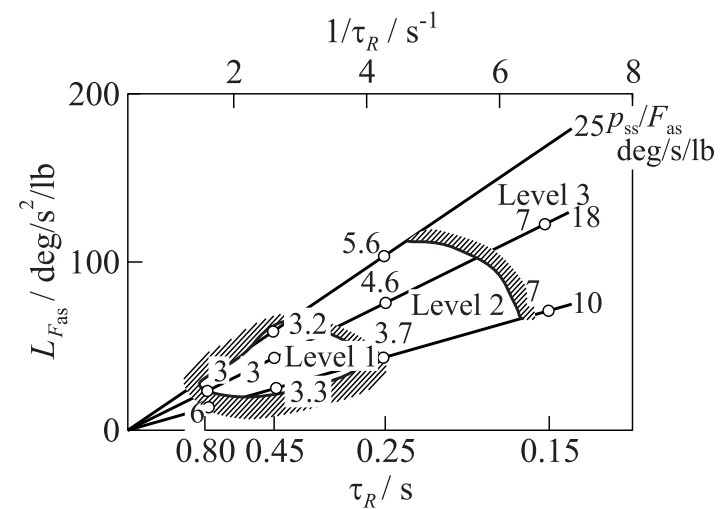

(b)

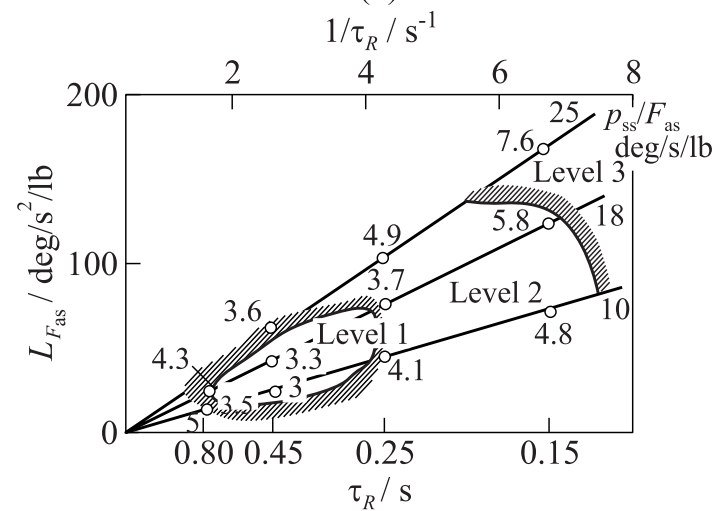

(c)

Figure 5 Comparison of McLATHOS $(a)$ and LATHOS (b) data [1] and estimations according to the TsAGI's method $(c)$ 


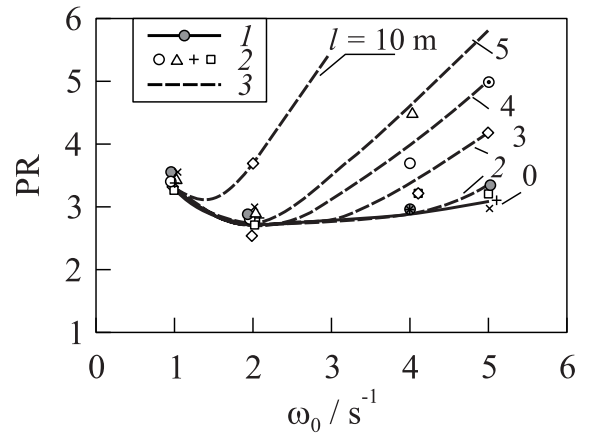

(a)

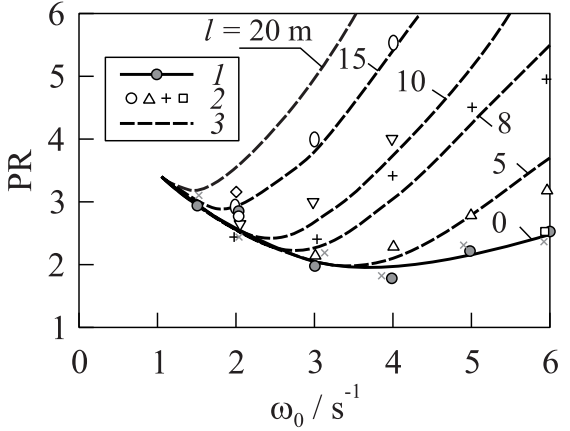

(b)

Figure 6 Pilot rating as a function of short-period mode frequency and pilot location relative to the center of gravity: $(a) n_{z \alpha}=5$ and $\delta_{n z}=-50 \mathrm{~mm} / \mathrm{g}$; and $(b) n_{z \alpha}=20$ and $\delta_{n z}=-20 \mathrm{~mm} / \mathrm{g} ; 1$ - fixed base; 2 - moving base; and 3 - criteria

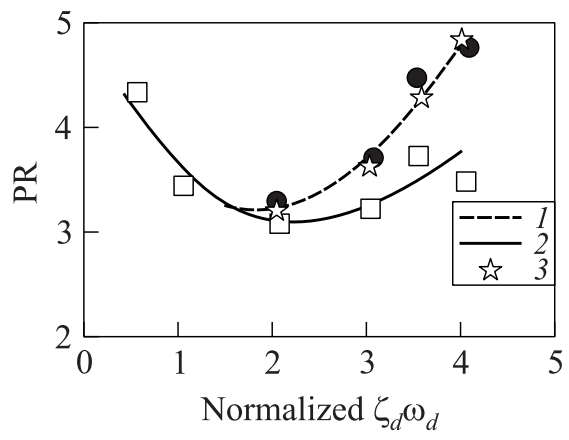

(a)

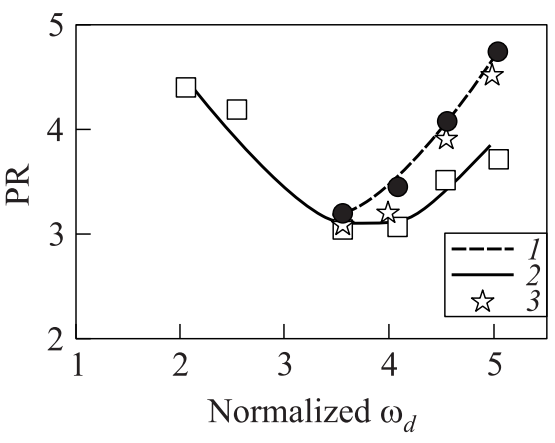

(b)

Figure 7 Effect of dutch roll damping $(1-$ motion on; $2-$ motion off; and $3-$ AR-criterion) and frequency on AR: $(a) \omega_{d}=0.7 \mathrm{~s}^{-1}$; and $(b) \xi_{d} \omega_{d}=0.4$

Figures 6 and 7 show a good agreement between the estimations and experimental data for longitudinal and directional control axes.

\subsection{Method to Correct Moving-Base Handling Qualities Results}

As it is mentioned in Introduction, the main distortions in moving-base experiments are caused by the cockpit drive algorithms. The relative pilot rating increment caused by the distortions can be estimated according to (1) where 
$\mathrm{PR}_{\text {motion }}$ is assumed to be the moving-base pilot rating provided there are no false acceleration cues. If they do arise, one has the following expression:

$$
\mathrm{PR}_{\text {motion } \Sigma}=\mathrm{PR}_{\text {motion }}+\Delta \mathrm{PR}_{\text {false cues }} \text {. }
$$

For moving-base case, pilot rating correction consists in estimating the inflight results $\mathrm{PR}_{\text {flight }}$ starting from the on-ground results $\mathrm{PR}_{\text {motion } \Sigma}$ available.

Expressions (1), (10), and (9) lead to the following:

$$
\begin{aligned}
& \mathrm{PR}_{\text {flight }} \\
& \quad=\mathrm{PR}_{\text {motion } \Sigma}+\left(\Delta \mathrm{PR}^{-}-\Delta \mathrm{PR}^{+}\right)\left(1-\Delta \mathrm{PR}_{\text {relative }}\right)-\Delta \mathrm{PR}_{\text {false cues }}
\end{aligned}
$$

where $\Delta \mathrm{PR}^{+}$and $\Delta \mathrm{PR}^{-}$are the pilot rating increments due to negative and beneficial acceleration effects calculated according to the method presented in section 2 .

Expression (11) is basic to translate moving-base pilot ratings into real flight conditions. Let consider a method to define the parameters $\Delta \mathrm{PR}_{\text {relative }}\left(\omega_{\mathrm{br}}, k\right)$ and $\Delta \mathrm{PR}_{\text {false cues }}$ in this expression.

It is known that the main methods to reproduce motion cues on the hexapodtype simulator are: high-pass filtering, scaling, and cockpit tilting to reproduce low-frequency lateral or longitudinal specific forces. In $[3,11,12]$, there are presented the criteria developed to estimate motion cueing fidelity as functions of the reproduction method, piloting task, and motion cues role in controlling of an aircraft. The criteria allow estimation of the relative pilot rating increment $\Delta \mathrm{PR}_{\text {relative }}\left(\omega_{\mathrm{br}}, k\right)$, caused by high-pass filtering and scaling, and the absolute pilot rating increment $\Delta \mathrm{PR}_{\text {false cues }}$ which arise while simulating the largeamplitude tasks.

The effect of high-pass filtering and scaling. It was shown in [3] that parameter $\Delta \mathrm{PR}_{\text {relative }}\left(\omega_{\mathrm{br}}, k\right)$ can be found as follows:

$$
\Delta \mathrm{PR}_{\text {relative }}\left(\omega_{\mathrm{br}}, k\right)=\Delta \mathrm{PR}_{\text {relative }}\left(\omega_{\mathrm{br}}\right) Q(k) .
$$

Parameter $\Delta \mathrm{PR}_{\text {relative }}\left(\omega_{\mathrm{br}}\right)$ is to introduce corrections for high-pass filtering effect and is determined from Fig. 8 for the cases of beneficial and negative effects.

High-pass filter cutoff frequency $\omega_{\mathrm{br}}$ has to satisfy the following condition:

$$
\left|Y_{\mathrm{hp}}\left(j \omega_{\mathrm{br}}\right)\right|=0.7 \text {. }
$$

Parameter $Q(k)$ in expression (12) is to introduce corrections for scaling. If the effect of accelerations is beneficial, parameter $Q(k)$ is determined from Fig. $9 a$, that is, from cockpit roll rate RMS, i. e., aircraft roll rate scaled down:

$$
\sigma_{p \operatorname{sim}}=k_{\phi} \sigma_{p \text { aircraft }}
$$




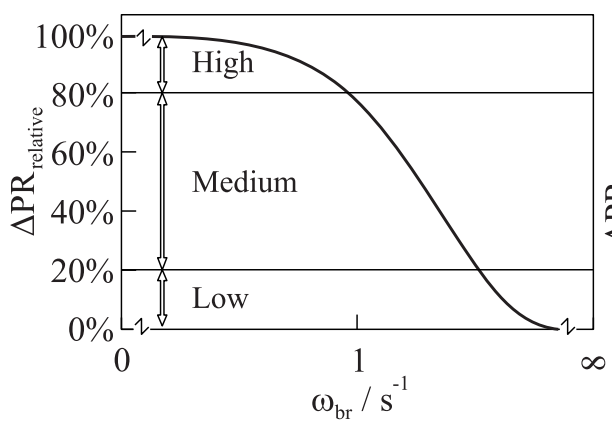

(a)

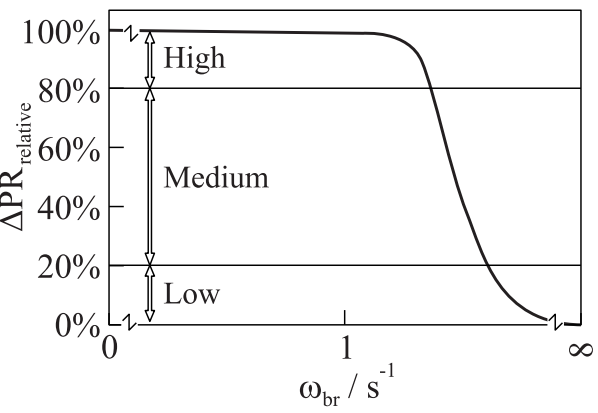

(b)

Figure 8 Motion fidelity as a function of high-pass filter break frequency: beneficial $(a)$ and negative $(b)$ acceleration effects

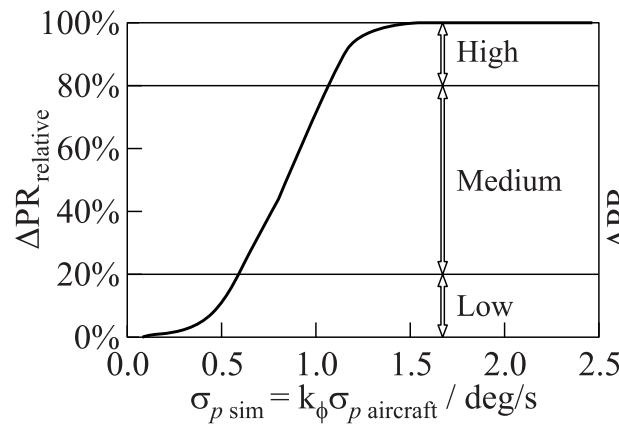

(a)

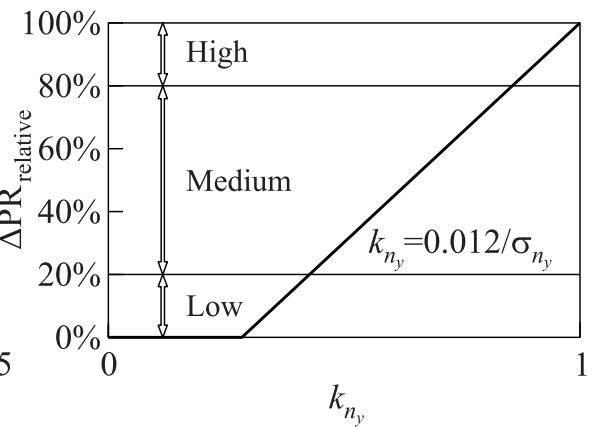

(b)

Figure 9 Motion fidelity criterion for scaling: beneficial $(a)$ and negative $(b)$ acceleration effects

If the effect of accelerations is negative, parameter $Q(k)$ is determined from Fig. $9 b$, that is, from lateral accelerations RMS and from the lateral accelerations scale: 100 percent acceleration simulation fidelity corresponds to $k_{n_{y}}=1$, while 0 percent acceleration simulation fidelity corresponds to the scale value when lateral accelerations decrease below their threshold values $\left(\sigma_{n_{y}}=0.012\right): k_{n_{y}}$ $=0.012 / \sigma_{n_{y}}$.

False motion cues effect. It was shown in $[3,11]$ that there are two types of false moiton cues: those due to cockpit tilting and the cues opposite in sign to aircraft acceleration cues. Unlike the latter, false specific forces due to cockpit tilting are practically impossible to compensate for, since the cockpit travel is 
insufficient for that. False specific forces arise simultaneously with opposite sign cues and usually suppress the perception of opposite sign cues. Thus, it is sufficient to take into account only the effect of false specific forces to correct the moving-base simulation results.

The effect of false specific forces on moving-base HQ results can be assessed from the curve in Fig. 10 if the values of the false cues are known.

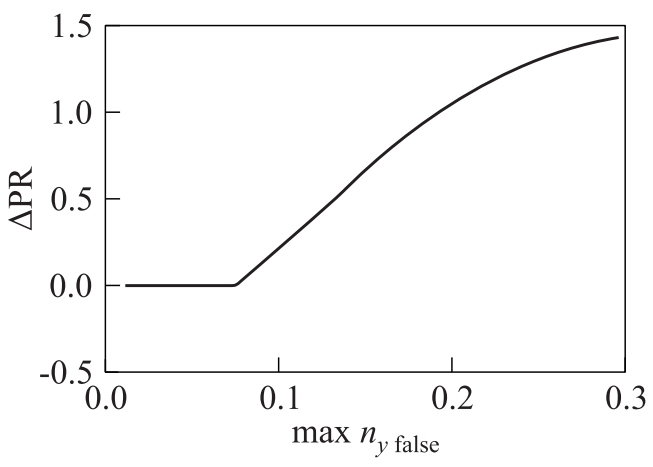

Figure 10 Criterion to estimate the effect of false specific forces due to cockpit tilting

\section{CONCLUDING REMARKS}

An integral approach is developed to adequately estimate and select handling qualities of an aircraft; it is based on the HQ criteria to assess the role of linear specific forces and angular accelerations on controlling the aircraft and on the criteria to assess the motion cueing fidelity.

The approach allows translation of the HQ pilot ratings received on on-ground simulators (both fixed-base and moving-base) into real-flight conditions, taking into account the role of accelerations on controlling the aircraft and distortions caused by motion system drive algorithms. The data received as a result of the translation are in a good agreement with the experimental data available (both on ground and in flight).

\section{REFERENCES}

1. Wood, J.R. 1983. Comparison of fixed-base and in-flight simulation results for lateral high order systems. AIAA Paper No. 83-2105.

2. Hess, R. A., and W. Siwakosit. 2001. Assessment of flight simulator fidelity in multiaxis tasks including visual cue quality. AIAA Paper No. 2001-4250.

3. Rodchenko, V.V., and A. D. White. 1999. Motion fidelity criteria based on human perception and performance. AIAA Paper No.99-4330.

4. Zaichik, L. E., V. V. Rodchenko, Yu. P. Yashin, and I. V. Rufov. 2000. A theoretical approach to estimation of acceleration effects on piloting. AIAA Paper No. 20004295 . 
5. Zaichik, L.E., V.V. Rodchenko, and Yu.P. Yashin. 2002. HQ criterion for roll control of high-augmented aircraft. AIAA Paper No. 2002-4801.

6. Zaichik, L. E., V. V. Rodchenko, I. V. Rufov, Y. I. Sneshko, and Y. P. Yashin. 2001. Method to estimate abrupt response of an aircraft in pitch control. Aviation Sci. Technol. LXXV(1). [In Russian.]

7. Zaichik, L. E., V. V. Rodchenko, Y. P. Yashin, and B. P. Lee. 2007. Abrupt response criteria for directional control. AIAA Paper No. 2007-6391.

8. Zaichik, L. E., V. V. Rodchenko, I. V. Rufov, Yu. P. Yashin, and A. D. White. 1999. Acceleration perception. AIAA Paper No. 99-4334.

9. McRuer, D. M., and E. Krendel. 1974. Mathematical models of human pilot. AGARD-AGD-188. $72 \mathrm{p}$.

10. Efremov, A. V., V.V. Rodchenko, A. V. Ogloblin, and A. N. Predtechensky. 1992. Pilot as a dynamic system. Moscow: Mashinostroyenie. [In Russian.]

11. Zaichik, L. E., Yu. P. Yashin, and P. A. Desyatnik. 2009. Motion fidelity criteria for large-amplitude tasks. AIAA Paper No. 2009-5916.

12. Zaichik, L.E., Yu.P. Yashin, and P.A. Desyatnik. 2010. Peculiarities of motion cueing for precision control tasks and maneuvers. ICAS. Nice, France. ICAS Paper No. 602 . 